\title{
Benefícios sobre a intensidade da dor, qualidade de vida e incapacidade de mulheres com dismenorreia submetidas a exercícios gerais versus método de Pilates: estudo-piloto
}

\author{
Benefits on pain intensity, quality of life, and disability of women with \\ dysmenorrhea undergoing general exercises versus Pilates: a pilot study
}

Bruna Cristina Taques Peruzzo ${ }^{1}$, Lisley Souza Ramalho ${ }^{1}$, Marta Regina Figueiredo², Fábio Marcon Alfieri ${ }^{3}$

${ }^{1}$ Curso de Graduação em Fisioterapia, Centro Universitário Adventista de São Paulo (UNASP) - São Paulo (SP), Brasil.

${ }^{2}$ Faculdades Metropolitanas Unidas (FMU) - São Paulo (SP), Brasil.

${ }^{3}$ Programa de Mestrado em Promoção da Saúde do UNASP - São Paulo (SP), Brasil.

DOI: http://dx.doi.org/10.7322/abcshs.v40i1.696

\begin{abstract}
RESUMO
Introdução: Dismenorreia primária é um distúrbio ginecológico caracterizado por dor relacionada à menstruação, sem causa patológica. Objetivo: Verificar e comparar a intensidade da dor, a incapacidade e a qualidade de vida em mulheres com dismenorreia primária submetidas a exercícios gerais versus Pilates. Métodos: Participaram do estudo 14 mulheres aleatorizadas em grupo de

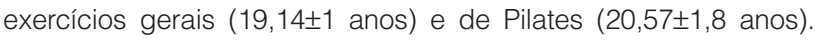
Ambos os grupos realizaram duas sessões por semana durante 50 minutos, no período de dois meses e meio, totalizando 20 sessões. O grupo de exercícios gerais realizou alongamentos e fortalecimentos dos músculos abdominais, lombares e assoalho pélvico. O grupo de Pilates, por sua vez, praticou exercícios para conscientização de pelve, com contrações do transverso e reto do abdômen, glúteos, períneo e eretores da coluna. As participantes responderam aos questionários de índice de incapacitação de Oswestry para dor lombar, Escala Visual Analógica (EVA), para medir a intensidade da dor e ao Questionário de Qualidade de Vida SF-36, antes e após a intervenção. Os dados foram considerados estatisticamente significantes quando $p<0,05$. Resultados: Foram encontradas melhoras significativas após a intervenção em ambos os grupos, em todas as avaliações. Em relação à diferença entre os grupos, na avaliação sobre a incapacitação para dor lombar, o grupo de exercícios gerais teve melhor resultado $(p<0,003)$ do que o grupo de Pilates, assim como no domínio de estado da saúde $(0,03)$ do Questionário SF-36. Conclusão: A prática de exercícios físicos gerais e Pilates por mulheres com dismenorreia podem reduzir a dor e a incapacidade e melhorar a qualidade de vida.
\end{abstract}

Palavras-chave: dismenorreia; exercício; dor; qualidade de vida; exercícios de alongamento muscular.

\begin{abstract}
Introduction: Primary dysmenorrhea is a gynecological disorder characterized by pain associated with menstruation without a pathological cause. Objective: To assess and compare pain intensity, disability and quality of life of women with primary dysmenorrhea undergoing general exercise versus Pilate's method. Methods: This study included 14 women randomly assigned to general exercise (19.14 \pm 1 years) group and Pilates group (20.57 \pm 1.8 years). Both groups performed two sessions per week for 50 minutes over two and a half months, totaling 20 sessions. The general exercise group performed stretching and strengthening of the abdominal and lower back muscles, and of pelvic floor. The Pilates group, on the other hand, practiced exercises for pelvis awareness with contractions of transverse and abdomen rectus muscles, buttocks, perineum and erectors of spine. All the participants responded to the questionnaires of the Oswestry disability index for low back pain; Visual Analogue Scale (VSA) to measure pain intensity; and SF-36 Quality of Life Questionnaire before and after the intervention. Data were considered statistically significant when $p<0.05$. Results: Significant improvements after the intervention in both groups were found in all evaluations. With regard to the differences between the groups, in the item of low back pain disability, the general exercise group had better outcomes $(p<0.003)$ than the Pilates group, as well as in the domain of health status (0.03) of the SF-36 Questionnaire. Conclusion: Practice of general exercises as well as Pilates's method by women with dysmenorrhea can reduce pain and disability and improve the quality of life.
\end{abstract}

Keywords: dysmenorrhea; exercise; pain; quality of life; muscle stretching exercises. 


\section{INTRODUÇÃO}

Considerada como um problema de saúde pública, a dismenorreia, que é um distúrbio ginecológico caracterizado por dor relacionada à menstruação, atinge cerca de $50 \%$ da população feminina pós-menarca ${ }^{1}$. É causa de absenteísmo às atividades laborais e de lazer, causando interrupções nas diversas práticas desenvolvidas diariamente ${ }^{2,3}$.

A dismenorreia é classificada em primária e secundária. A primária ocorre com maior incidência na adolescência, não existe uma anormalidade estrutural visível ou patológica. Geralmente, a dor do tipo cólica é sentida sobre o abdômen inferior e região lombossacral. Quando a sensação é muito forte, a mulher pode apresentar episódios de vômitos e náuseas, seguidos ou não de diarreia. A dismenorreia provém do istmo uterino hipertônico, resultando em uma retenção temporária de resíduos do fluxo menstrual, causando grande pressão nesta área. A dor pode estar associada à isquemia em função da vigorosa contração dos músculos do útero. Já a dismenorreia secundária extrínseca ou adquirida, pode ser decorrente de alguma patologia pélvica que pode ser responsabilizada pela dor menstrual. As causas mais frequentes são: endometriose, miomas, pólipos endometriais e lesões causadas pelo uso do dispositivo intrauterino, doença inflamatória pélvica ou problemas de fertilidade ${ }^{3-6}$.

Dentre os tratamentos para esse tipo de condição clínica está a prática de exercícios físicos, que pode ser uma opção a fim de se conseguir diminuição da dor e melhora da qualidade de vida destas mulheres.

A prática de exercícios gerais, os quais envolvem alongamentos, fortalecimentos e mobilizações, pode interferir positivamente na dismenorreia. Um estudo realizado com universitárias mostrou que mulheres ativas apresentam menor intensidade de dor em relação às inativas ${ }^{7}$, e isto ocorre, pois o exercício físico pode contribuir para a melhor adequação do metabolismo, do equilíbrio hidroeletrolítico e do aporte sanguíneo na região pélvica, além de fatores como o controle da dor pelo melhor equilíbrio de neurotransmissores que inibem a dor ${ }^{8-10}$.

Uma opção de exercício físico pode ser a utilização de alguns métodos como o de Pilates, que recebe este nome devido ao seu criador Joseph Pilates (1880-1967), e que nos últimos anos se popularizou e é praticado tanto na reabilitação quanto no fitness. Os exercícios de Pilates têm como ênfase o controle do corpo, a posição e o movimento e podem ser realizados no solo ou em equipamentos especializados, porém todos seguem os princípios tradicionais que incluem centralização, concentração, controle, precisão e respiração. As atividades visam desenvolver consciência e equilíbrio corporal, além de coordenação motora e mobilidade das articulações ${ }^{11}$. Embora os estudos sobre a aplicabilidade do método estejam em seus primórdios, acredita-se que devido às suas características de foco na conscientização corporal, objetivando força, estabilidade postural, flexibilidade, controle muscular e respiração $0^{11}$, este possa ser um recurso a ser utilizado na dismenorreia. Como ainda são escassos os estudos a respeito da dismenorreia e a prática de exercícios físicos ${ }^{7}$ o método Pilates pode ser uma opção. $\mathrm{O}$ objetivo deste estudo foi comparar a qualidade de vida, a intensidade da dor e a incapacidade de mulheres com dismenorreia submetidas ao método Pilates versus os exercícios gerais.

\section{MÉTODOS}

\section{Amostra}

Trata-se de um ensaio clínico prospectivo, no qual 14 mulheres participaram por meio de convites por escrito e verbal. Todas eram estudantes universitárias e foram divididas aleatoriamente, por meio de sorteio simples, em dois grupos de intervenção: exercícios gerais e Pilates. Todas eram nulíparas e deveriam apresentar dismenorreia primária e possuir sintomas há mais de um ano. Não participaram do estudo mulheres que estivessem praticando atividades físicas regulares, apresentassem diagnóstico de dismenorreia secundária, utilizassem medicamentos antiespasmódicos durante o período de intervenção e apresentassem sangramento vaginal irregular. Todas elas foram informadas detalhadamente sobre os procedimentos utilizados e concordaram em participar de maneira voluntária do estudo, assinando um Termo de Consentimento Livre e Esclarecido.

A pesquisa foi conduzida dentro dos padrões éticos exigidos pela Declaração de Helsinque de 1964 e em conformidade com a resolução 196/96 do Conselho Nacional de Saúde. Os procedimentos do estudo foram aprovados pelo Comitê de Ética do Centro Universitário Adventista de São Paulo, sob o protocolo 110/2010.

\section{Instrumentos e procedimentos}

As participantes responderam ao questionário de qualidade de vida SF-36, que se trata de um questionário multidimensional formado por 36 itens para avaliar a qualidade de vida, incluindo oito componentes: capacidade funcional (dez itens), aspectos físicos (quatro itens), dor (dois itens), estado geral de saúde (cinco itens), vitalidade (quatro itens), aspectos sociais (dois itens), aspectos emocionais (três itens), saúde mental (cinco itens) e mais uma questão de avaliação comparativa entre as condições de saúde atual e de um ano atrás. Ele analisa tanto aspectos negativos de saúde (doença ou enfermidade), como positivos (bem-estar). Os dados são avaliados a partir da transformação das respostas em escores em uma escala de 0 a 100, de cada componente, não havendo um único valor que resuma toda a avaliação, resultando em um estado geral de saúde melhor ou pior ${ }^{12}$.

Também foi aplicado o Questionário Oswestry, que examina as incapacidades relacionadas à coluna lombar, a qual quantifica o grau de incapacidade. Ele contém 10 perguntas, cujas respostas podem pontuar de zero a cinco e, posteriormente, o somatório dos pontos é multiplicado por dois a fim de que seja obtida uma pontuação percentual, na qual quanto maior a pontuação, maior a incapacidade ${ }^{13}$.

A intensidade da dismenorreia foi determinada por meio da Escala Visual Analógica (EVA) ${ }^{14}$, que se constitui em uma reta de $10 \mathrm{~cm}$ traçada sobre um papel. Em uma das extremidades está escrito "sem dor" e na outra, "máximo de dor". Foi solicitado a cada mulher que marcasse sobre a reta um sinal indicando seu nível de desconforto dentro da EVA. Quanto mais próxima da origem fosse a marcação $(0 \mathrm{~cm})$, menor seria o nível de dor percebido. Por outro lado, quanto mais próximo do final da reta $(10 \mathrm{~cm})$, maior o nível de dor percebido. 
Ambos os grupos realizaram todas as avaliações antes e após os programas de exercícios.

A intervenção foi realizada na Policlínica do Centro Universitário Adventista de São Paulo. O protocolo teve duração de dois meses e meio, totalizando 20 sessões, que ocorriam duas vezes por semana, com duração de 50 minutos cada.

O grupo de exercícios gerais praticou exercícios convencionais indicados para a dismenorreia, que envolviam: báscula, anteversão e retroversão da pelve, nos quais a mobilidade articular da cintura pélvica é abordada. Foram realizados alongamentos e fortalecimentos dos músculos abdominais, lombares e assoalho pélvico nas posturas: em pé, sentada e deitada, associados à respiração.

Para o grupo de Pilates, foram realizados dez exercícios para a conscientização de pelve em posição neutra, nos quais a voluntária era orientada a contrair os grupos musculares do cinturão pélvico: transverso e reto do abdômen, glúteos, assoalho pélvico e eretores da coluna. Todos os exercícios aplicados tiveram como princípio a conscientização do cinturão pélvico. O método Pilates propicia a realização de exercícios de modo harmônico, com poucas repetições e associados à respiração. As atividades transcorreram do nível básico ao intermediário, objetivando aumentar o grau de dificuldade dos exercícios, promovendo uma melhora da força muscular, do alongamento, do equilíbrio, da amplitude de movimento e da postura adequada. Os exercícios foram realizados nas posturas em pé, sentada na bola, deitada no colchonete, deitada em decúbito dorsal e ventral sobre a bola.

Os exercícios sempre foram realizados de forma que fossem agradáveis e com a finalidade de não causar dor ou gerar tensões e sobrecargas.

\section{Análise estatística}

A análise considerou um estudo com braços paralelos e $80 \%$ de probabilidade de se detectar uma diferença de tratamento, com nível de significância de 5\%. Caso a diferença verdadeira entre os tratamentos fosse de três pontos na EVA, acrescentávamos dois indivíduos em cada grupo a fim de compensar possíveis perdas, totalizando 14 indivíduos. A análise dos dados foi realizada em pacote estatístico InStat do fabricante GraphPad Software, Inc. As informações estão apresentadas em médias e desvios padrão e passaram pelo teste de KolmogorovSmirnov para verificação da normalidade. As comparações entre os grupos antes da intervenção foram feitas com o teste $t$ não pareado. As comparações dentro de cada grupo após a intervenção foram encontradas com o uso do teste $t$ pareado. A comparação de seu efeito entre os grupos foi feita com o uso do teste $t$ para dados não pareados. O nível de significância considerado foi de $5 \%(\mathrm{p}<0,05)$.

\section{RESULTADOS}

Fizeram parte deste estudo 14 pacientes, com média de idade de $23 \pm 3$ anos e tempo de queixa de dismenorreia de $8,1 \pm 2$ anos. $\mathrm{Na}$ Tabela 1 observa-se que os grupos de programas de exercícios eram similares quanto à idade e composição corporal.

Com relação à intensidade da dor, houve diminuição após os programas de intervenção em ambos os grupos. Quanto à qualidade de vida, esta foi melhorada também nos dois grupos, não sendo um superior ao outro nas duas avaliações. No entanto, cabe ressaltar que, ao avaliar os oito domínios sobre a qualidade de vida, em seis houve melhora no grupo que realizou exercícios gerais, ao passo que o mesmo se deu em apenas três domínios no grupo de Pilates. Apenas o domínio estado de saúde apresentou melhoria significante do grupo de exercícios gerais em relação ao de Pilates. Sobre a incapacidade, embora ambos os grupos tenham diminuído, aquele que realizou exercícios gerais teve aprimoramento superior comparado ao de Pilates, conforme mostra a Tabela 2.

\section{DISCUSSÃO}

Atualmente, existem vários recursos para o tratamento dos sintomas da dismenorreia, no entanto mesmo afetando a qualidade de vida destas adolescentes, somente cerca de $15 \%$ procura uma consulta médica a fim de discutir o problema ${ }^{15}$. A intensidade da dor, a incapacidade e a qualidade de vida das mulheres com dismenorreia submetidas tanto ao método Pilates quanto aos exercícios gerais foram investigadas neste estudo, o qual constatou mudanças positivas nestes aspectos após a execução dos exercícios.

A questão sobre exercícios físicos e a dismenorreia vem sendo investigada há muito tempo. Estudos de $1943^{16}, 1966^{17}, 1969^{18}$ e $1971^{19}$ vêm tentando relacionar estes dois assuntos. Em 1998, ainda havia escassez de estudos que fornecessem evidências sólidas dos benefícios do exercício sobre a dismenorreia, continuando ainda em $2008^{20}$.

O estudo de Jahormi, Gaeini e Rahimi ${ }^{21}$, embora não tenha sido aleatorizado, reporta que a prática regular durante 12 semanas de exercícios cardiovasculares com componentes de treino muscular, de agilidade e velocidade, durante 90 minutos com frequência de duas sessões semanais, pode influenciar positivamente em relação aos sintomas físicos e psíquicos de mulheres com dismenorreia.

Quanto à prática de exercícios físicos para a dismenorreia, Brown e Brown ${ }^{22}$, após realizarem revisão de estudos controlados randomizados comparados com Grupo Controle, observaram que ainda há um baixo número de estudo e, dos quatro selecionados pelos autores, apenas um com amostra pequena e com algumas falhas metodológicas pôde ser usado. Em uma investigação que comparou $^{23}$ mulheres que realizaram programa de treino cardiovascular durante 12 semanas com o Grupo Controle, verificou-se diferença a respeito da diminuição dos sintomas. Recentemente, um estudo controlado composto por 92 voluntárias randomizadas em Grupo Controle e grupo de exercícios de Yoga relatou que tanto a severidade da dor, quanto a sua intensidade são diminuídas em mulheres que praticaram algumas posturas de tal atividade ${ }^{24}$.

Tabela 1: Características gerais da amostra dos grupos exercícios gerais e Pilates

\begin{tabular}{|l|c|c|c|}
\hline Variáveis & Exercícios gerais & Pilates & Valor $\mathbf{p}$ \\
\hline $\mathrm{n}$ & 7 & 7 & \\
\hline Idade $(\mathrm{anos})$ & $19,14 \pm 1$ & $20,57 \pm 1,8$ & $0,10^{*}$ \\
\hline IMC $\left(\mathrm{kg} / \mathrm{cm}^{2}\right)$ & $20,4 \pm 1,04$ & $22,6 \pm 1,66$ & $0,12^{*}$ \\
\hline
\end{tabular}

IMC: índice de massa corpórea, *teste $t$ não pareado 
Tabela 2: Resultados das diferentes avaliações clínicas entre os grupos - exercícios gerais e Pilates, antes e após os programas de exercícios, e comparação dos efeitos das intervenções entre os grupos

\begin{tabular}{|c|c|c|c|c|c|c|c|}
\hline \multirow[b]{2}{*}{ Variáveis } & \multicolumn{3}{|c|}{ Exercícios gerais } & \multicolumn{3}{|c|}{ Pilates } & \multirow{2}{*}{$\begin{array}{c}\text { Valor } p \\
\text { Grupo } \\
\text { versus tempo }\end{array}$} \\
\hline & Antes & Depois & Valor $\mathbf{p}$ & Antes & Depois & Valor $p$ & \\
\hline EVA $(\mathrm{cm})$ & $7,14 \pm 1,50$ & $1,80 \pm 1,06$ & 0,0001 & $7,50 \pm 0,90$ & $2,0 \pm 1,15$ & 0,0001 & 0,3 \\
\hline Oswestry & $10,28 \pm 1,90$ & $2,70 \pm 1,60$ & 0,004 & $5,57 \pm 6,70$ & $2,1 \pm 3,3$ & 0,05 & 0,03 \\
\hline SF-36 (total) & $63,87 \pm 10,37$ & $78,62 \pm 7,46$ & 0,002 & $72,37 \pm 7,96$ & $83,75 \pm 12,12$ & 0,004 & 0,2 \\
\hline Capacidade funcional & $75,0 \pm 17,92$ & $92,85 \pm 6,42$ & 0,01 & $87,14 \pm 10,97$ & $84,28 \pm 30,75$ & 0,40 & 0,14 \\
\hline Aspectos físicos & $67,85 \pm 31,94$ & $81,42 \pm 17,87$ & 0,05 & $82,14 \pm 22,01$ & $89,28 \pm 18,01$ & 0,28 & 0,65 \\
\hline Dor & $62,28 \pm 17,11$ & $78,14 \pm 7,21$ & 0,03 & $65,42 \pm 15,07$ & $71,71 \pm 21,96$ & 0,20 & 0,37 \\
\hline Estado de saúde & $54,85 \pm 12,20$ & $68,14 \pm 12,89$ & 0,0006 & $65,57 \pm 8,74$ & $65,28 \pm 16,13$ & 0,47 & 0,03 \\
\hline Vitalidade & $65,71 \pm 13,20$ & $77,85 \pm 15,08$ & 0,01 & $67,14 \pm 13,32$ & $78,57 \pm 11,86$ & 0,03 & 0,92 \\
\hline Aspectos sociais & $71,42 \pm 21,87$ & $79,35 \pm 17,28$ & 0,15 & $71,71 \pm 20,78$ & $89,42 \pm 10,39$ & 0,05 & 0,43 \\
\hline Aspectos emocionais & $42,71 \pm 38,72$ & $81,40 \pm 16,14$ & 0,01 & $71,37 \pm 37,54$ & 100,0 & 0,05 & 0,63 \\
\hline Saúde mental & $68,57 \pm 10,56$ & $71,42 \pm 7,22$ & 0,34 & $69,14 \pm 15,67$ & $79,42 \pm 8,66$ & 0,08 & 0,44 \\
\hline
\end{tabular}

EVA: escala visual analógica; SF-36: questionário de qualidade de vida

A prática regular de exercícios gerais pode trazer melhoras referentes à dismenorreia provavelmente em função da melhor adequação do metabolismo, maturação dos órgãos pélvicos e extrapélvicos, equilíbrio hidroeletrolítico e aporte sanguíneo à região pélvica ${ }^{7,8}$. Por envolverem esta região, os mesmos podem ter permitido uma irrigação sanguínea mais abundante, promovendo uma massagem nos órgãos internos, deixando-os relaxados ${ }^{25}$.

Os exercícios praticados pelo método Pilates também demonstraram ser positivos em relação à dor, funcionalidade e qualidade de vida. Há forte evidência de que o método possa melhorar a flexibilidade e o equilíbrio dinâmico e uma evidência moderada para resistência muscular $^{26}$. Estudo recente ${ }^{27}$ comprova os benefícios do método Pilates realizado no solo e com bolas, durante 10 sessões, quanto à diminuição dos sintomas e melhora da dor em mulheres com dismenorreia. Embora não tenha havido comparação com outro tipo de exercícios como nosso estudo, esses resultados corroboram os nossos referentes ao método Pilates, que também mostrou ser útil para diminuir a intensidade da dor, melhorar a funcionalidade e a qualidade de vida.

Avaliando mulheres com pouca ou nenhuma disfunção da musculatura do assoalho pélvico submetidas ao treinamento de força muscular versus método de Pilates, notou-se que ambas as técnicas foram capazes de melhorar a força, não sendo um superior ao outro. Os autores reportam que mais estudos devem ser realizados para determinar se o método Pilates pode realmente tratar a disfunção do assoalho pélvico ${ }^{28}$. As conclusões obtidas no presente estudo também não confirmam que o método Pilates provê melhores resultados do que outro tipo de exercícios, no caso os gerais. Estes, por sua vez, foram superiores aos de Pilates em relação à melhora da incapacidade analisada pelo questionário de Oswestry, que engloba atividades como dormir, ter vida social e sexual, levantar, caminhar, dentre outras. No entanto, cabe ressaltar que, antes da intervenção, os valores do grupo de exercícios gerais eram maiores do que os de Pilates. Neste estudo, foi escolhido o instrumento "Índice de Incapacidade de Oswestry”, pois além de ser validado para a língua portuguesa, é muito usado em estudos internacionais e mostra excelentes níveis de validade e confiabilidade ${ }^{13}$, além do fato que a dor lombar está presente em até $80 \%$ das mulheres com dismenorreia ${ }^{27}$, o que certamente influencia as atividades cotidianas, detectadas por este questionário ${ }^{13}$.
Um fator analisado nesta investigação foi a qualidade de vida, que foi aprimorada após a participação nos grupos de intervenção. Acreditamos que isto pode ter sido possibilitado pois os exercícios terapêuticos podem propiciar diversos benefícios fisiológicos e psicológicos e estão relacionados a alterações bioquímicas envolvidas com a liberação de neurotransmissores, com a ativação de receptores específicos e a adequação dos níveis serotoninérgicos ${ }^{29}$, o que pode ter interferido positivamente na melhor percepção de qualidade vida dessas jovens. Essa percepção é importante e também têm sido avaliada por meio do SF-36 por outros autores, os quais utilizaram tal instrumento para analisar a qualidade de vida em mulheres com dismenorreia ${ }^{30,31}$. Ressalta-se neste estudo que, ao avaliar os domínios, estes foram melhorados em seis dos oito no grupo que realizou exercícios gerais, ao passo que somente três o fizeram no grupo de Pilates. Porém, ao comparar o efeito da intervenção entre os grupos, apenas o estado de saúde foi estatisticamente superior no grupo de exercícios gerais, mostrando que este tipo é benéfico para o estado de saúde em geral de mulheres com dismenorreia.

Acredita-se que métodos como o de Pilates, assim como exercícios gerais, proporcionem resultados positivos em função da contribuição de melhor oxigenação tecidual, equilíbrio hidroeletrolítico e liberação de substâncias que fornecem bem-estar, como os opioides endógenos e a endorfina, em resposta aos exercícios ${ }^{7,9,10}$.

No entanto, mesmo tomando os apropriados cuidados quanto à alocação dos voluntários, intervenção e avaliação, um fator limitante neste estudo foi a amostra reduzida. Por isso, estudos com amostras maiores são desejáveis com a utilização deste e de outros tipos de exercícios com metodologias apropriadas, a fim de que seja aumentada a evidência científica sobre a melhoria do quadro clínico da dismenorreia em indivíduos praticantes de exercícios físicos.

Conclui-se que tanto o grupo de exercícios gerais quanto o método de Pilates apresentaram melhoras significantes com relação à qualidade de vida, capacidade funcional e diminuição da intensidade da dor em mulheres com dismenorreia. Porém, por se tratar de um estudo-piloto, o alcance dos resultados merece ser visto com cautela, e novos estudos com amostras maiores são desejáveis a fim de replicarmos ou até mesmo encontrarmos diferentes conclusões. 


\section{REFERÊNCIAS}

1. Osayande AS, Mehulic S. Diagnosis and initial management of dysmenorrhea. Am Fam Physician. 2014;89(5):341-6.

2. Proctor ML, Farquhar CM. Dysmenorrhoea. BMJ Clin Evid. 2007;2007:0813

3. Proctor M, Farquhar C. Diagnosis and management of dysmenorrhoea. BMJ. 2006;332(7550):1134-8. http://dx.doi.org/10.1136/bmj.332.7550.1134

4. Sahin S, Ozdemir K, Unsal A, Arslan R. Review of frequency of dysmenorrhea and some associated factors and evaluation of the relationship between dysmenorrhea and sleep quality in university students. Gynecol Obstet Invest. 2014;78(3):179-85. http://dx.doi.org/10.1159/000363743

5. Durain D. Primary dysmenorrhea: assessment and management update. J Midwifery Womens Health. 2004;49(6):520-8. http://dx.doi.org/10.1016/j.jmwh.2004.08.013

6. Diegoli MS, Diegoli CA. Dismenorréia. RBM. 2007;64(3):81-7

7. Quintana LM, Heinz LN, Portes LA, Alfieri FM. Influência do nível de atividade física na dismenorréia. Rev Bras Ativ Fis Saúde. 2010;15(2):101-4.

8. Ikeda F, Salomão AJ, Ramos LO. Dismenorréia primária. RBM. 1999;56(12):215-25

9. Souza JB. Poderia a atividade física induzir analgesia em pacientes com dor crônica? Rev Bras Med Esporte. 2009;15(2):145-50. http://dx.doi.org/10.1590/S1517-86922009000200013

10. Mcardle WD, Katch Fl, Victor L. Fisiologia do exercício: energia, nutrição e desempenho humano. 6 ed. Rio de Janeiro: Guanabara Koogan; 2008. p. 695

11. Wells C, Kolt GS, Bialocerkowski A. Defining Pilates exercise: a systematic review. Complement Ther Med. 2012;20(4):253-62. http://dx.doi.org/10.1016/j.ctim.2012.02.005

12. Ciconelli RM, Ferraz MB, Santos W. Tradução para a língua portuguesa e validação do questionário genérico de avaliação de qualidade de vida SF-36 (Brasil SF-36). Rev Bras Reumatol. 1999;39(3):143-50.

13. Vigatto R, Alexandre NM, Correa Filho HR. Development of a Brazilian Portuguese version of the Oswestry Disability Index: cross-cultural adaptation, reliability, and validity. Spine. 2007:32(4):481-6.

http://dx.doi.org/10.1097/01.brs.0000255075.11496.47

14. Chapman CR, Syrjala KL. Assessment of pain. In: Bonica JJ, Chapman CR, Fordyce WE, Loeser JD, eds. The management of pain in clinical practice. 2 ed. Philadelphia: Lea \& Febiger; 1990. p. 580-94.

15. Sultan C, Gaspari L, Paris F. Adolescent dysmenorrhea. Endocr Dev. 2012:22:171-80. http://dx.doi.org/10.1159/000331775

16. Billig Jr HE. Dysmenorrhoea: the result of a postural defect. Arch Surg. 1943:46(5):611-3. http://dx.doi.org/10.1001/archsurg.1943.01220110027007
17. Sebek V, Lewit K. [Indications for exercise and neuro-orthopedic therapy in cases of menstrual pain]. Cesk Gynekol. 1966;31(10):754-5.

18. House JM. Report on exercises for dysmenorrhoea. Occup Health (Lond). 1969;21(1):31-4.

19. Timonen S, Procopé BJ. Premenstrual syndrome and physical exercise. Acta Obstet Gynecol Scand. 1971;50(4):331-7.

20. Daley AJ. Exercise and primary dysmenorrhoea: a comprehensive and critical review of the literature. Sports Med. 2008:38(8):659-70.

21. Jahromi MK, Gaeini A, Rahimi Z. Influence of a physical fitness course on menstrual cycle characteristics. Gynecol Endocrinol. 2008;24(11):659-62.

http://dx.doi.org/10.1080/09513590802342874

22. Brown J, Brown S. Exercise for dysmenorrhoea. Cochrane Database Syst Rev. 2010;7(2):CD004142. http://dx.doi.org/10.1002/14651858.CD004142.pub2

23. Israel R, Sutton M, O'Brien K. Effects of aerobic training on primary dysmenorrhoea symptomology in college females. J Am Coll Health. 1985;33(6):241-4.

24. Rakhshaee Z. Effect of three yoga poses (cobra, cat and fish poses) in women with primary dysmenorrhea: a randomized clinical trial. J Pediatr Adolesc Gynecol. 2011;24(4):192-6. http://dx.doi.org/10.1016/j.jpag.2011.01.059

25. Cornélio AM, Siqueira VP, Botelho SP, Branco M. Estudo comparativo entre a estimulação elétrica nervosa transcutânea e cinesioterapia na analgesia em pacientes com dismenorreia primária. Fisio Brasil. 2006;10(76):27-33.

26. Cruz-Ferreira A, Fernandes J, Laranjo L, Bernardo LM, Silva A. A systematic review of the effects of pilates method of exercise in healthy people. Arch Phys Med Rehabil. 2011;92(12):2071-81. http://dx.doi.org/10.1016/j.apmr.2011.06.018

27. Araújo LM, Silva JM, Bastos WT, Ventura PL. Diminuição da dor em mulheres com dismenorreia primária, tratadas pelo método Pilates. Rev Dor. 2012;13(2):119-23. http://dx.doi.org/10.1590/S1806-00132012000200004

28. Culligan PJ, Scherer J, Dyer K, Priestley JL, Guingon-White G, Delvecchio $\mathrm{D}$, et al. A randomized clinical trial comparing pelvic floor muscle training to a Pilates exercise program for improving pelvic muscle strength. Int Urogynecol J. 2010;21(4):401-8. http://dx.doi.org/10.1007/s00192-009-1046-z

29. Sonenreich C, Kerr CF, Estevão G. Doenças afetivas. São Paulo: Manole; 1991

30. Unsal A, Ayranci U, Tozun M, Arslan G, Calik E. Prevalence of dysmenorrhea and its effect on quality of life among a group of female university students. Ups J Med Sci. 2010;115(2):138-45. http://dx.doi.org/10.3109/03009730903457218

31. Barnard K, Frayne SM, Skinner KM, Sullivan LM. Health status among women with menstrual symptoms. J Womens Health (Larchmt). 2003;12(9):911-9. http://dx.doi.org/10.1089/154099903770948140 